\title{
RECONSTRUCCIÓN MICROQUIRÚRGICA EN EL TRAUMA PEDIÁTRICO*
}

\author{
Drs. Andrea Hasbún N. ${ }^{1}$, Silvana Acosta V. ${ }^{1,2}$, \\ Patricio Andrades C. ${ }^{3}$, Juan Pablo Cisternas V. ${ }^{3}$ \\ 1 Equipo de Cirugía Plástica. Hospital de Niños Roberto del Río. \\ 2 División de Cirugía Plástica. Escuela de Medicina, Pontificia Universidad Católica de Chile. \\ 3 Departamento de Cirugía. Hospital Clínico de la Universidad de Chile. \\ Santiago, Chile.
}

\begin{abstract}
Microsurgical reconstruction in pediatric trauma

Introduction: We present a series of clinical cases in which free tissue transfer was performed to repair traumatic defects in 4 pediatric patients ( 5 free flaps). Each clinical case was analyzed for free flap indication, complication and outcome. Case reports: There were a total of 2 musculotaneous flaps (latissimus dorsi and rectus abdominis) and 3 fasciocutaneous flaps (anterolateral thigh). One case required surgical revision and no flap losses are reported. At present patients are in rehabilitation phase after an average of 12 months of follow-up. This series is the first national communication of microsurgical reconstruction in the pediatric trauma patient.
\end{abstract}

Key words: Microsurgery, children, trauma, reconstructive surgery, free flap.

\section{Resumen}

Introducción: Se presenta una serie de casos clínicos en la cual se realiza transferencia libre de tejidos para solucionar defectos de cobertura de origen traumático en 4 pacientes pediátricos ( 5 colgajos libres). Se analiza cada caso clínico, la indicación de colgajo libre, complicaciones y resultados. Casos clínicos: Se realizaron 2 colgajos musculares (dorsal ancho y recto abdominal) y 3 colgajos fasciocutáneos (anterolateral de muslo). Sólo un caso requirió revisión quirúrgica, no tuvimos pérdidas de colgajos y hasta la fecha los pacientes se encuentran en fase de rehabilitación, tras un seguimiento medio de 12 meses. Esta serie es la primera comunicación nacional de reconstrucción microquirúrgica en pacientes pediátricos traumáticos.

Palabras clave: Microcirugía, niños, trauma, cirugía reconstructiva, colgajo libre.

\footnotetext{
*Recibido el 4 de mayo de 2015 y aceptado para publicación el 29 de mayo de 2015.

Los autores no refieren conflictos de interés.

Correspondencia: Dra. Silvana Acosta V. contacto@doctoraacosta.cl
} 


\section{Introducción}

La reconstrucción microquirúrgica de los defectos de cobertura es una técnica ampliamente usada en el mundo entero. A medida que las técnicas microquirúrgicas se han ido desarrollando, la complejidad y extensión de los defectos que podemos reparar ha ido en aumento, logrando incluso llevar tejido compuesto ${ }^{1-4}$. En niños, la técnica se consideró un verdadero reto para el cirujano plástico, por el pequeño calibre de los vasos y la tendencia al vaso espasmo ${ }^{5}$; sin embargo, la incorporación de mejor instrumental y la consolidación de la curva de aprendizaje microquirúrgica han redundado en la optimización de resultados obtenidos en niños ${ }^{6,7}$.

En Chile los equipos con mayor experiencia en este tipo de reconstrucción se concentran en hospitales de pacientes adultos (mutuales de seguridad u oncológicos). El sistema de salud público nacional, no tiene implementada la posibilidad de manejo agudo del trauma pediátrico que requiera microcirugía. La experiencia local en esta área es aún muy escasa, son sólo grupos colaborativos del extrasistema o reportes aislados orales, no hay publicaciones nacionales de reconstrucción microquirúrgica en niños.

El propósito de esta comunicación es mostrar una serie de casos de transporte libre de tejidos en pacientes menores de 15 años con lesiones de origen traumático, analizando cada caso clínico, su indicación, complicaciones y resultado funcional.

\section{Pacientes y Métodos}

Se revisó, en forma retrospectiva, los casos clínicos consecutivos con defectos severos de cobertura provocados por trauma grave, en los que se practicó reconstrucción con colgajo microquirúrgico. Todos los pacientes fueron manejados por los autores entre noviembre de 2012 y marzo de 2014. Se indicó cobertura con colgajo libre considerando el tamaño del defecto y el compromiso de estructuras nobles como hueso, vasos o nervios. Se analizó la indicación de microcirugía, la permeabilidad vascular, las complicaciones, el tiempo quirúrgico y el resultado funcional. Por lo limitado de la serie sólo se utilizó sólo estadística descriptiva.

\section{Resultados}

Serie de 4 pacientes en los cuales se realizaron 5 colgajos libres. Tres hombres y 1 mujer, con un rango etario entre los 4 y 11 años. Cuatro casos presentaron lesiones en extremidad inferior y un caso en la extremidad superior (Tabla 1). En cada caso se analizó la necesidad de cobertura según el tamaño del defecto, el compromiso de estructuras nobles, la condición de los tejidos locales y la condición general del paciente por lesiones asociadas al trauma grave.

En el primer caso se utilizaron 2 colgajos, dorsal ancho (DA) y recto abdominal (RA), en los casos 2, 3 y 4 el colgajo de elección fue el anterolateral de muslo (ALT). El tiempo quirúrgico varió entre

Tabla 1. Características clínicas de la serie

\begin{tabular}{|c|c|c|c|c|c|c|}
\hline Casos & Edad & Sexo & Mecanismo & $\begin{array}{c}\text { Zona } \\
\text { afectada }\end{array}$ & Lesión & $\begin{array}{l}\text { Diagnósticos } \\
\text { asociados }\end{array}$ \\
\hline 1 & 11 & $\mathrm{~F}$ & Atropello & $\begin{array}{c}\text { Pierna } \\
\text { derecha }\end{array}$ & $\begin{array}{l}\text { Fractura expuesta tibia der IIIa* } \\
\text { bilateral } \\
\text { Conminución severa }\end{array}$ & $\begin{array}{l}\text { TEC leve } \\
\text { Shock hipovolémico } \\
\text { Sepsis por hongos }\end{array}$ \\
\hline 1 & 11 & $\mathrm{~F}$ & Atropello & $\begin{array}{l}\text { Pierna } \\
\text { izquierda }\end{array}$ & $\begin{array}{l}\text { Fractura expuesta tibio-peronea } \\
\text { izquierda IIIa* }\end{array}$ & Idem \\
\hline 2 & 7 & M & Explosión & $\begin{array}{c}\text { Antebrazo } \\
\text { derecho }\end{array}$ & $\begin{array}{l}\text { Herida compleja muñeca derecha } \\
\text { Pérdida de segmento nervio mediano } \\
\text { Amputación falange distal dedo medio } \\
\text { e índice }\end{array}$ & $\begin{array}{l}\text { TEC abierto } \\
\text { Exposición vísceras } \\
\text { Fractura mandibular } \\
\text { Quemadura vía aérea } \\
\text { Trauma ocular }\end{array}$ \\
\hline 3 & 4 & M & Atropello & $\begin{array}{c}\text { Pie } \\
\text { izquierdo }\end{array}$ & $\begin{array}{l}\text { Sección de tendón extensor del Hallux, } \\
\text { fracturas no desplazadas de metáfisis } \\
\text { distal de peroné y de cuña lateral }\end{array}$ & \\
\hline 4 & 11 & M & Atropello & $\begin{array}{l}\text { Pierna } \\
\text { izquierda }\end{array}$ & Fractura expuesta IIIa* & \\
\hline
\end{tabular}

*Clasificación de Gustilo para fracturas expuestas. 
Tabla 2. Resultados quirúrgicos de la serie

\begin{tabular}{|c|c|c|c|c|c|}
\hline & Paciente 1 & Paciente 2 & Paciente 3 & Paciente 4 & Paciente 5 \\
\hline Colgajo utilizado & LD & RA & ALT & ALT & ALT \\
\hline Vasos receptores & $\begin{array}{c}\text { Tibiales } \\
\text { posteriores }(\mathrm{T}-\mathrm{T})\end{array}$ & $\begin{array}{c}\text { Tibiales } \\
\text { posteriores }(\mathrm{T}-\mathrm{T})\end{array}$ & $\begin{array}{l}\text { Radiales } \\
\text { (T-L) }\end{array}$ & $\begin{array}{c}\text { Tibiales } \\
\text { posteriores (T-T) }\end{array}$ & $\begin{array}{l}\text { Peroneos } \\
\text { (T-T) }\end{array}$ \\
\hline Momento efectuado & $\begin{array}{c}20 \text { días } \\
\text { postaccidente }\end{array}$ & $\begin{array}{c}27 \text { días } \\
\text { postaccidente }\end{array}$ & $\begin{array}{c}14 \text { días } \\
\text { postaccidente }\end{array}$ & $\begin{array}{c}15 \text { días } \\
\text { postaccidente }\end{array}$ & $\begin{array}{c}12 \text { días } \\
\text { postaccidente }\end{array}$ \\
\hline Tiempo quirúrgico & $450 \mathrm{~min}$ & $380 \mathrm{~min}$ & $310 \mathrm{~min}$ & $420 \mathrm{~min}$ & $490 \mathrm{~min}$ \\
\hline Anticoagulantes & $\begin{array}{l}\text { Dalteparina } \\
\text { sódica }\end{array}$ & $\begin{array}{l}\text { Dalteparina } \\
\text { sódica }\end{array}$ & No & $\begin{array}{l}\text { Dalteparina } \\
\text { sódica }\end{array}$ & No \\
\hline $\begin{array}{l}\text { Monitorización } \\
\text { colgajo }\end{array}$ & Clínica & Clínica & Clínica & Doppler & Clínica \\
\hline $\begin{array}{l}\text { Complicaciones } \\
\text { locales }\end{array}$ & No & No & $\begin{array}{l}\text { Trombosis } \\
\text { venosa }\end{array}$ & $\begin{array}{c}\text { Infección y } \\
\text { dehiscencia zona } \\
\text { dadora }\end{array}$ & No \\
\hline Reoperaciones & No & No & $\mathrm{Si}$ & No & No \\
\hline Alta & $\begin{array}{c}39 \text { días post } \\
\text { segunda cirugía }\end{array}$ & $\begin{array}{l}39 \text { días post } \\
\text { cirugía }\end{array}$ & $\begin{array}{l}27 \text { días después } \\
\text { de reexploración }\end{array}$ & $\begin{array}{c}10 \text { días } \\
\text { postcirugía }\end{array}$ & $\begin{array}{l}14 \text { días } \\
\text { postcirugía }\end{array}$ \\
\hline
\end{tabular}

LD: Latissimus dorsi. RA: Recto anterior. ALT: Anterolateral de muslo. T-T: Término-terminal. T-L: Término-lateral.

5 y 9 h. Los vasos receptores en 3 colgajos fueron los tibiales posteriores. En todos los colgajos la monitorización fue principalmente clínica y sólo en un caso se complementó con doppler. La estadía preoperatoria fue entre 12 y 27 días y la postoperatoria fue entre 10 y 39 días. No se presentó ninguna falla total de la anastomosis y sólo hubo una falla parcial por trombosis venosa que requirió re-ex- ploración, logrando su rescate en forma exitosa con puente venoso (Tabla 2). El tiempo de seguimiento promedio fue de 12 meses (rango 4 a 22). Hasta el seguimiento actual, los pacientes se encuentran sin complicaciones, con cobertura estable y reintegrados a su vida normal. Desde la Figura 1 a la 5 se pueden ver imágenes pre y postoperatorias de cada uno de los colgajos utilizados.
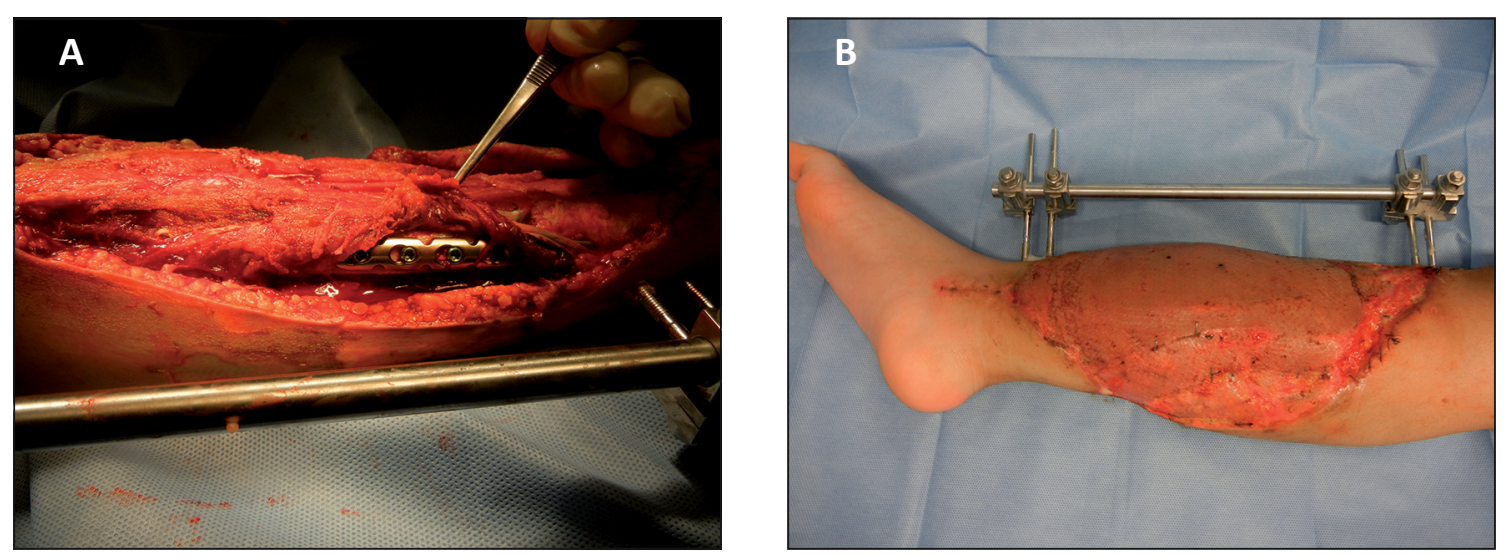

Figura 1. Caso clínico 1. Colgajo M. Dorsal Ancho. Paciente femenino 11 años, atropellada en Antofagasta; A) Fractura expuesta pierna derecha con material de osteosíntesis sin cobertura; B) Control postoperatorio 20 días de colgajo libre Dorsal Ancho. 

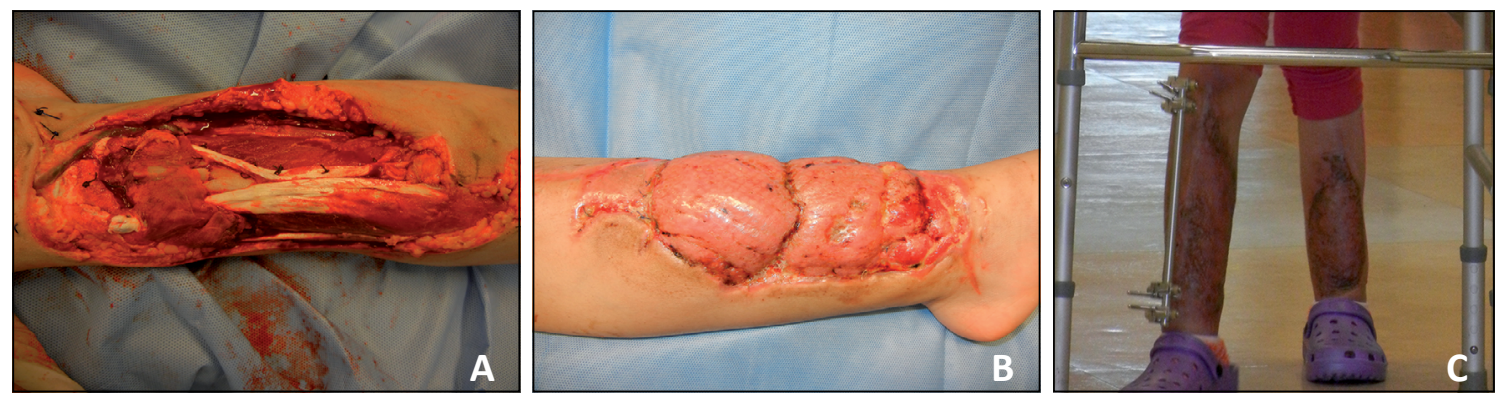

Figura 2. Caso clínico 1. Colgajo M. Recto abdominal. A) Lesión en pierna izquierda exposición ósea y tendones; B) Reconstrucción con colgajo Recto abdominal libre, más injerto de piel; C) Control 3 meses postoperatorio en rehabilitación.
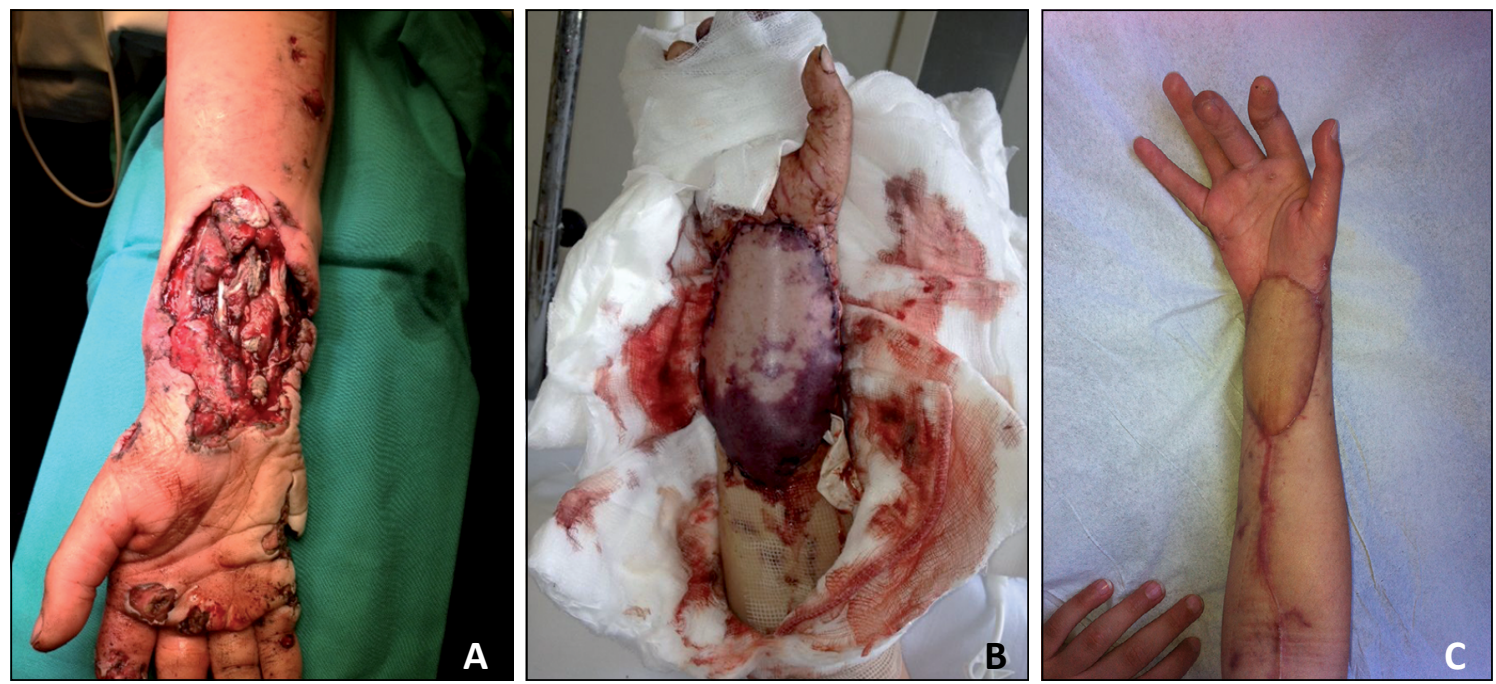

Figura 3. Caso clínico 2. Paciente masculino de 7 años, manipulación de aparato explosivo. A) Defecto muñeca derecha con exposición del aparato flexor y sección de N. Mediano; B) Cobertura con colgajo ALT, sufrimiento parcial por trombosis venosa a las 48 h. C) Evolución del colgajo ALT tras reexploración y rescate.
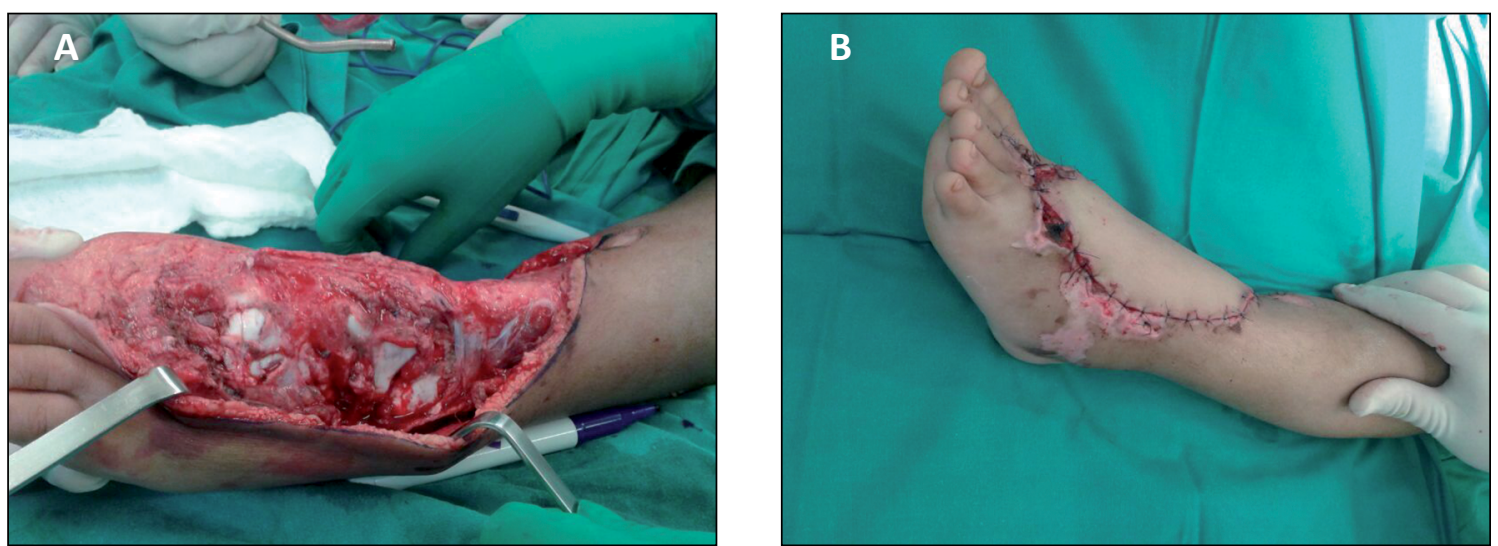

Figura 4. Caso clínico 3. Paciente masculino de 4 años, atropello en vía pública; A) Defecto de cobertura en dorso del pie izquierdo con exposición de estructuras óseas y tendíneas; B) Cobertura con colgajo ALT al tercer día postoperatorio. 

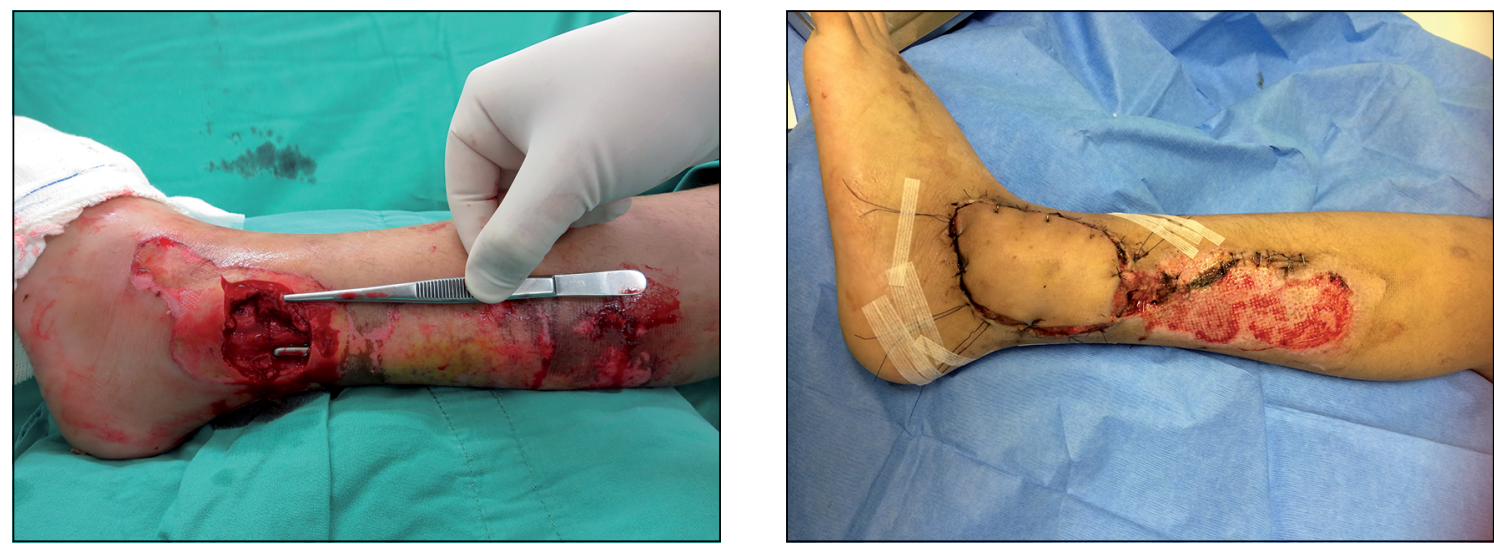

Figura 5. Caso clínico 4. A) Fractura expuesta no desplazada distal de peroné. La pinza muestra extensión de todo el bolsillo proximal subcutáneo; B) Cobertura con colgajo ALT, evolución al $4^{\circ}$ día postoperatorio.

\section{Discusión}

La microcirugía en niños se desarrolló desde mediados de los años 70, algo posterior al inicio de la microcirugía en adultos. Harii la reporta como técnicamente difícil por el tamaño de los vasos y una alta tendencia al vaso espasmo ${ }^{8}$. Actualmente, la experiencia publicada es cuantitativamente menor que la de la población adulta pero con un alto porcentaje de éxito, alrededor del $98 \%$ con vasos de calibre superior a $0,7 \mathrm{~mm}^{9}$. Las recomendaciones de autores más experimentados son usar siempre instrumental microquirúrgico bajo alta magnificación, disección cuidadosa de los vasos, uso abundante de vasodilatadores locales, control del dolor postoperatorio y prevención del vaso espasmo con el uso de ferulización de la extremidad ${ }^{10}$. En el grupo pediátrico, el precepto de la escalera reconstructiva debe permanecer sólo como orientación general para estos defectos complejos, ya que la microcirugía es la herramienta adecuada que nos permite un tratamiento óptimo; y en la gran mayoría de los casos es la primera elección ${ }^{11,12}$.

Esta serie constituye la primera experiencia de trabajo sistemático en el manejo de las lesiones traumáticas graves en niños del sistema público, donde se ha podido ofrecer el estándar de oro para el manejo de estas complejas lesiones traumáticas. Se utilizaron colgajos clásicos de anatomía conocida y de amplia difusión en cirugía plástica. En cada caso, el colgajo se seleccionó según la extensión del defecto, el tipo de tejido requerido y las condiciones del paciente. Los pacientes ingresan en el contexto de trauma severo por lo que existen lesiones asociadas y complicaciones de las mismas que dilatan la posibilidad de cobertura precoz. El tiempo entre el accidente y la intervención disminuyó progresiva- mente, probablemente debido a mayor difusión de la existencia de este recurso (Tabla 1). El colgajo más utilizado fue el ALT y el tiempo quirúrgico varió en un rango de 5 a $9 \mathrm{~h}$, comparable a la experiencia en adultos y a otras series de pacientes pediátricos ${ }^{13}$. La monitorización de los colgajos fue clínica en la mayoría de los casos y sólo se re-exploró un paciente con falla parcial del colgajo, lográndose rescate exitoso con puente venoso.

En la evaluación de si la técnica microquirúrgica fue o no apropiada para manejar los casos seleccionados, es importante establecer si los colgajos son capaces de cumplir con su propósito. Si bien el tiempo de seguimiento es corto (promedio de 12 meses), hasta ahora los 4 casos presentan una cobertura estable, con capacidad de deambulación y reintegro a su actividad habitual. En esta serie, los colgajos fueron elegidos principalmente por las características del defecto. En relación a los colgajos musculares, la disección no presentó dificultades y en los colgajos de perforantes, a pesar del pequeño tamaño de sus vasos, se logró una adecuada visualización y disección con lupa de aumento 3,5x. La anastomosis se realizó siempre bajo microscopio.

La transferencia libre de tejido en niños, usualmente no presenta los factores de riesgo que sí enfrentamos en los adultos como son: el tabaquismo, y la enfermedad vascular periférica lo que por sí solo debe traducirse en una mejor posibilidad de éxito en este grupo, si sumado a esto, cuidamos los aspectos antes mencionados en relación al manejo de los vasos, no debiera asombrarnos las buenas cifras de éxito publicadas internacionalmente ${ }^{14,15}$. Sin embargo, hay temas que merecen una consideración especial y aún no han sido totalmente analizados: el efecto del crecimiento y desarrollo en el tejido trasplantado, así como las repercusiones psicológicas 
secundarias y la discapacidad a tan temprana edad de la zona dadora ${ }^{16}$. Es aconsejable que los centros de atención infantil tengan protocolos de manejo y derivación a centros de referencia ya que la larga curva de aprendizaje y el complejo montaje del escenario microquirúrgico pueden mejorar cuando se ha logrado un trabajo sistemático, coordinado y continuo.

\section{Referencias}

1. Yucel A, Aydin Y, Altintas F, Senyuva C. Elective freetissue transfer in pediatric patients. J Reconstr Microsurg. 2001;17:27-36.

2. Chiang YC, Jeng SF, Yeh MC, Lui YT, Chen HT, Wei FC. Free tissue transfer for leg reconstruction in children. Microsurgery 1991;12:207-15.

3. Hidalgo DA, Disa JJ, Cordeiro PG, Hu QY . A review of 716 consecutive free flaps for oncologic surgical defects: refinement in donor-site-selection and technique. Plast Reconstr Surg. 1998;102:722-32.

4. Van Beek AL, Wavak PW, Zook EG. Microvascular surgery in young children. Plast Reconstr Surg. 1979;63:457-62.

5. Duteille F, Lim A, Dautel G. Free flap coverage of upper and lower limb tissue defects in children: a series of 22 patients. Ann Plast Surg. 2003;50:344-9.

6. Parry SW, Toth BA, Elliot LF. Microvascular free-tissue transfer in children. Plast Reconstr Surg. 1988;81:83840.
7. Yildirim S, Calikapan GT, Akoz T. Reconstructive microsurgery in pediatric population-a serie of 25 patients. Microsurgery 2008;28:99-107.

8. Harii K, Ohmori K. Free groin flaps in children. Plast Reconstr Surg. 1975;55:588-59.

9. Gilbert A. Reconstruction of congenital hand defects with microvascular toe transfers. Hand Clin. 1985;1:351-60.

10. Devaraj VS, Kay SP, Batchelor AG, Yates A. Microvascular surgery in children. British J Plastic Surg. 1991;44:276-80.

11. Hong JP. The use of supermicrosurgery in lower extremity reconstruction: the next step in evolution. Plas Reconstruc Surg. 2009;123:230-5.

12. Khouri RK, Shaw WW. Reconstruction of the lower extremity with microvascular free flaps: a 10-year experience with 304 consecutive cases. J Trauma 1989;108694.

13. Konttila E, Koljonen V, Kauhanen S, Kallio P, Tukiainen E. Microvascular reconstruction in children. Journal of Trauma 2010;68:548-52.

14. Aboelatta YA, Aly HM. Free tissue transfer and replantation in pediatric patients: technical feasibility and outcome in a series of 28 patients. J Hand Microsurg. 2013;5:74-80.

15. Van Landuyt K, Hamdi M, Blondeel P, Tonnard P, Verpaele A, Monstrey S. Free perforator flaps in children. Plast Reconstr Surg. 2005;116:159-69.

16. Shenaq SM, Dihn TA. Pediatric microsurgery. Reconstruction by free tissue transfer. Clin Plast Surg. 1990;17:85-94. 